\title{
Immunoregulatory effects of glioma-associated stem cells on the glioblastoma peritumoral microenvironment: a differential PD-L1 expression from core to periphery?
}

\author{
*Grazia Menna, MD, Ivana Manini, PhD, ${ }^{2}$ Daniela Cesselli, MD, PhD, ${ }^{2}$ Miran Skrap, MD, ${ }^{3}$ \\ Alessandro Olivi, MD, ${ }^{1}$ Tamara lus, MD, PhD, ${ }^{3}$ and Giuseppe Maria Della Pepa, MD1 \\ ${ }^{1}$ Institute of Neurosurgery, Fondazione Policlinico Universitario Agostino Gemelli IRCCS, Catholic University, Rome; ${ }^{2}$ Institute \\ of Pathology, University Hospital, Udine; and ${ }^{3}$ Neurosurgery Unit, Department of Neuroscience, Santa Maria della Misericordia, \\ University Hospital, Udine, Italy
}

\begin{abstract}
OBJECTIVE Glioma-associated stem cells (GASCs) have been indicated as possible players in supporting growth and recurrence in glioblastoma. However, their role in modulating immune response in the peritumoral area has not yet been described. In this study, the authors aimed to investigate programmed death-ligand 1 (PD-L1) differential expression at the protein level in GASCs derived from different tumor areas (core, periphery, and surrounding healthy brain).

METHODS Tumor tissue samples were collected from patients who underwent surgery for a histopathologically confirmed diagnosis of glioblastoma. Sampling sites were confirmed via neuronavigation and categorized on 5-aminolevulinic acid (5-ALA) fluorescence as bright (ALA+), pale (ALA PALE), or negative (ALA-), which corresponds to the tumor mass, infiltrated peritumoral area, and healthy brain, respectively, during surgery. GASCs were first isolated from the 3 regions and analyzed; then Western blot analysis was used to evaluate the level of PD-L1 expression in the GASCs.

RESULTS Overall, 7 patients were included in the study. For all patients, the mean values \pm SD of PD-L1 expression in GASCs for ALA+, ALA PALE, and ALA- were $1.12 \pm 1.14,0.89 \pm 0.63$, and $0.57 \pm 0.18$, respectively. The differentially expressed values of PD-L1 in GASCs sampled from the 3 areas were found to be significant $(p<0.05)$ for 3 of the 7 patients: patient S470 (ALA+ vs ALA- and ALA PALE vs ALA-), patient S473 (ALA+ vs ALA PALE and ALA PALE vs $A L A-)$, and patient S509 (ALA+ vs ALA-).

CONCLUSIONS This analysis showed, for the first time, that GASCs expressed a constitutive level of PD-L1 and that PD-L1 expression in GASCs was not uniform among patients or within the same patient. GASC analysis combined with 5-ALA-guided sampling (from core to periphery) made it possible to highlight the role of the tumor microenvironment at the infiltrating margin, which might cause clinical resistance, opening interesting perspectives for the future.

https://thejns.org/doi/abs/10.3171/2021.11.FOCUS21589
\end{abstract}

KEYWORDS glioblastoma; immunotherapy; PD-1; PD-L1; 5-ALA; glioma-associated stem cells

$\mathrm{T}$ HE most common and aggressive malignant, primary brain tumor is glioblastoma (GBM); more than 12,000 new cases are diagnosed each year in Europe. ${ }^{1,2}$ Disentangling its complexity is challenging,,$^{3,4}$ as GBM exhibits a diffuse invasion pattern in which tumor cell infiltration makes complete resection virtually impossible and subsequent treatments, including radiotherapy, chemotherapy, and immunotherapy, unsuccessful in eradicating the tumor. The reason behind the failure of these therapies is not yet well known, but several hypotheses have been put forward. First, infiltrating cells are enriched with glioma stem cells, a minor subpopulation of selfrenewing malignant cells that maintain a low but steady level of unlimited proliferation and are characterized by chemotherapy refractiveness. ${ }^{5,6}$ To explain the failure of immunotherapy, the "seed or soil" approach is gaining ground. In this case, "seed" indicates tumor subtypes and their intrinsic characteristics, while "soil" indicates the tumor microenvironment (TME), which interacts with the "seed" and whose contribution seems to be critical in this setting. ${ }^{7-13}$

To prove this, the clinical need for therapies targeting

ABBREVIATIONS $\mathrm{ALA}+=$ tumor core area (bright 5-ALA fluorescence); $A L A-=$ tumor outer periphery area (no 5-ALA fluorescence); ALA PALE = tumor inner periphery area (pale 5-ALA fluorescence); GASC = glioma-associated stem cell; GBM = glioblastoma; IOD = integrated optical density; $\mathrm{PD}-1=$ programmed cell death protein 1 ; PD-L1 = programmed death-ligand 1; TME = tumor microenvironment; 5-ALA = 5-aminolevulinic acid.

SUBMITTED October 1, 2021. ACCEPTED November 15, 2021.

INCLUDE WHEN CITING DOI: 10.3171/2021.11.FOCUS21589.

${ }^{*}$ G.M. and I.M. share first authorship of this work. T.I. and G.M.D.P. share senior authorship of this work. 


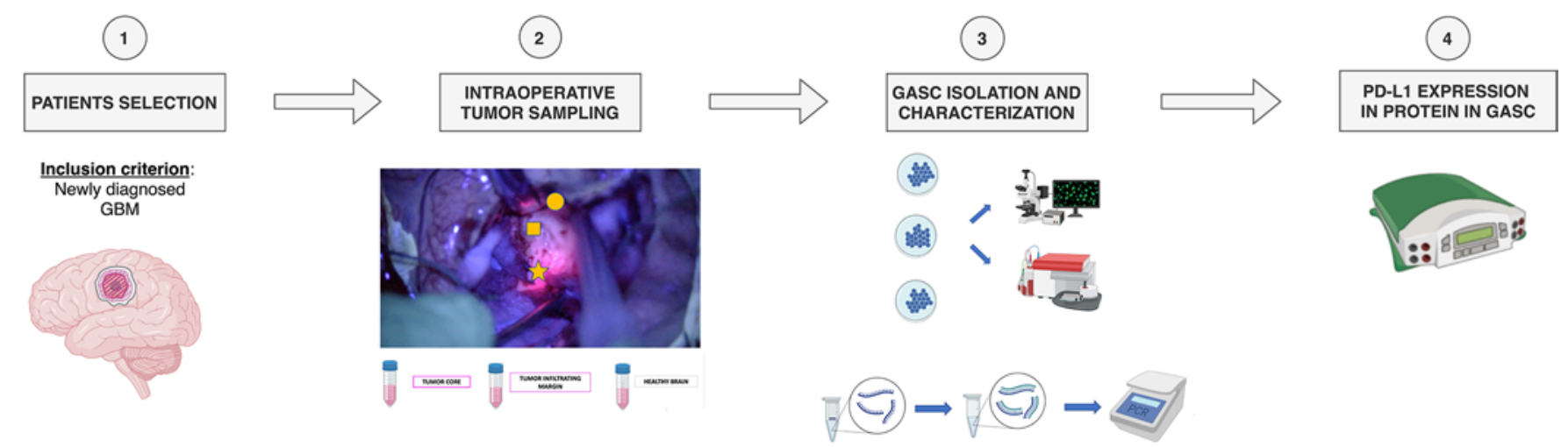

FIG. 1. Schematic representation of our methodology. 1: Tumor tissue samples were collected from patients who underwent surgery for a histopathologically confirmed diagnosis of GBM. 2: Sampling sites were assessed via neuronavigation (classified as ALA+ [star], ALA PALE [square], and ALA- [circle]). 3: GASCs were effectively isolated and the third passage was reached in culture; then the GASCs were detached and underwent flowcytometry and immunofluorescence, before being analyzed via polymerase chain reaction. 4: Western blotting was used to evaluate the level of PD-L1 expression in GASCs isolated from the 3 regions. The level of PD-L1 expression was evaluated by densitometric analyses for each cell line and calculated after normalization of vinculin expression.

the remaining tumor cell population has been coupled with the discovery of the TME's role in promoting survival, invasion, and proliferation. In GBM, the TME consists of all nonmalignant elements present in the tumor that maintain, support, or hinder its evolution; it can be regarded as "plastic soil" due to its ability to change the functions of those elements based on environmental stimuli. There are 3 known unique features of the GBM TME. 1) The bloodbrain barrier, even after losing integrity in the context of tumor progression, remains impermeable to most chemotherapeutics. 2) Myelinated and interconnected axon tracts have the role of providing haptotactic cues to drive cellular invasion and represent a major barrier to surgical resection itself. 3) A distinct extracellular matrix and stromal cell composition are present, the latter of which is notable for its relationship to T-cell disfunction, myeloid cells as mediators of tumor progression, and dendritic and natural killer T cells. ${ }^{14}$ In addition, stem cells interact with the TME and their interplay is mandatory for biological resistance and to sustain the tumorigenic process. Against this background, our group previously isolated a subpopulation of stem cells, called glioma-associated stem cells (GASCs). These cells might represent a patient-based model of the TME, as they are not characterized by tumor-initiating properties while still maintaining the ability to support the biological aggressiveness of the tumor. ${ }^{15-17}$

Against this background, the role of checkpoint inhibitors is not of secondary importance. Recently, it was demonstrated that the negative regulation of $\mathrm{T}$ cells was mediated by the programmed cell death protein 1 (PD-1) pathway. PD-1 is expressed predominantly by activated T lymphocytes and is often activated by programmed deathligand 1 (PD-L1), a ligand that is known to be expressed by antigen-presenting cells, parenchymal cells, and $\mathrm{B}$ lymphocytes. Importantly, PD-L1 expression has been detected in glioma; studies have shown its heterogeneity in GBM tumor mass, with greater expression seen at the edges of the tumor than in the core. This last point has led to the analysis of regulatory mechanisms contributing to enriched PD-L1 expression in the peritumoral microenvironment. 18,19

To date, PD-L1 expression in GASCs has not yet been described; therefore, our study aimed to investigate its differential expression at the protein level in GASCs derived from different GBM areas (core, periphery, and surrounding healthy brain), both between patients and within the same patient. To achieve this goal, we performed a targeted sampling of 3 areas characterized by different 5-aminolevulinic acid (5-ALA) fluorescence-bright (ALA+), pale (ALA PALE), and negative (ALA-) - corresponding to the tumor mass, infiltrated peritumoral area (beyond the area of enhancement on MRI), and the healthy brain, respectively, during surgery. ${ }^{20-24}$

\section{Methods}

The independent ethics committees of both the Azienda Ospedaliero-Universitaria of Udine (Parere 196/2014/ Em) and the Fondazione Policlinico Universitario Agostino Gemelli (Prot. No. 0020786/18) approved this study. Written informed consent was obtained from all patients, and clinical investigations were conducted according to the principles expressed in the Declaration of Helsinki. Our methodology is summarized in Fig. 1.

\section{Patient Selection}

Samples were collected from patients who underwent surgery for a histopathologically confirmed diagnosis of GBM in the neurosurgical departments of the Azienda Ospedaliera Universitaria of Udine and the Fondazione Policlinico Universitario Agostino Gemelli IRCCS (Rome, Italy). Exclusion criteria included needle biopsies, recurrent gliomas, and secondary surgeries. All patients were treatment naive, and none underwent any neoadjuvant treatment. For each patient, demographics (sex and age at diagnosis), radiological data (tumor location, side, midline shift, ependymal and corpus callosum involvement, presence of necrosis/cystic area, tumor volume [measured on 

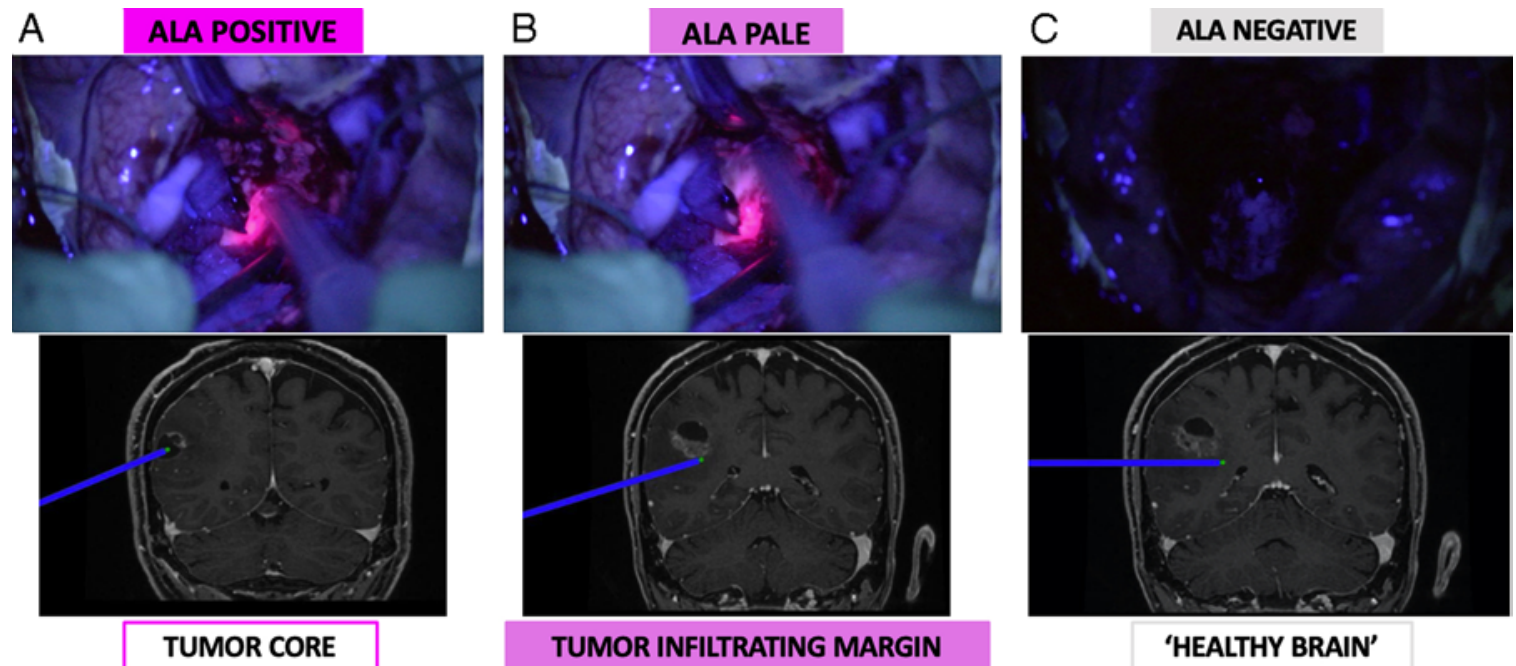

FIG. 2. Sampling areas as seen under violet-blue light $(370-440 \mathrm{~nm}$ ) exhibiting 5-ALA fluorescence (upper row) matched with the images obtained from the intraoperative navigation system (lower row). A: ALA+ area corresponding to the tumor core. B: ALA PALE area corresponding to the tumor infiltrating margin. C: ALA- area corresponding to the macroscopically healthy brain.

contrast-enhanced T1-weighted MRI], and extent of resection), molecular data (O-6-methylguanine-DNA methyltransferase methylation, isocitrate dehydrogenase $1[I D H 1]$ mutation, and Ki-67 proliferative index), and clinical data (postsurgical oncological protocol) were collected.

\section{Intraoperative Tumor Sampling}

Three to 5 hours before surgery, 5-ALA $(20 \mathrm{mg} / \mathrm{kg}$ of body weight; Medac $\mathrm{GmbH}$ ) was administered orally to patients. During surgery, 3 different tumor sites were visualized based on the presence of red-violet fluorescence, following excitation with ultraviolet blue light (FL 400 filter, Leica M720 OH5, Leica Microsystems).

Sampling sites were confirmed via intraoperative neuronavigation and classified as follows: 1) The ALA+ area is analogous to the enhancing area in neuronavigation and corresponds to the tumor core, with lava-like red fluorescence. 2) The ALA PALE area is analogous to the tumoral boundaries surrounding the enhancing area and corresponds to the tumor inner periphery, with pale red fluorescence. 3) The tumor outer periphery, without fluorescence, is the ALA- area and is analogous to the nonenhancing areas distant from the tumor (Fig. 2).

For each sampling site, a few cubic millimeters of tissue was collected and preserved in DMEM (Invitrogen) at $4^{\circ} \mathrm{C}$ before processing.

\section{GASC Isolation and Characterization}

GASCs were efficiently isolated from all 3 sampling sites, showing a progressive intensity of fluorescence for 5-ALA. The GASCs were then maintained, in vitro, applying a previously established protocol that has been optimized for culturing multipotent adult stem cells from normal and neoplastic human tissues. ${ }^{24}$

\section{PD-L1 Expression in the Protein of GASCs}

Whole-cell extract proteins were obtained by cell ly- sis in radioimmunoprecipitation assay (RIPA) buffer for 40 minutes on ice, followed by centrifugation at $10,000 \mathrm{~g}$ for 15 minutes at $4^{\circ} \mathrm{C}$, and then the protein-enriched supernatants were collected. The protein concentration was measured by bicinchoninic acid protein assay (Euroclone) following the manufacturer's instructions. Next, $25 \mu \mathrm{g}$ of proteins was resolved on sodium dodecyl sulfate-polyacrylamide gel electrophoresis (SDS-PAGE), transferred, and immobilized on a $0.45-\mu \mathrm{M}$ nitrocellulose membrane (Amersham plc). Membranes were blocked by incubation for 1 hour at room temperature, with 5\% bovine serum albumin in Tris-buffered saline (TBS; Tris- $\mathrm{HCl} 50 \mathrm{mM}$, $\mathrm{pH} 7.4$, and $\mathrm{NaCl} 150 \mathrm{mM}$ ) containing $0.2 \%$ Tween 20 and hybridized, overnight at $4^{\circ} \mathrm{C}$ with rabbit monoclonal to PD-L1 (E1L3N) XP antibody (Cell Signaling Technology, Inc.) and rabbit polyclonal to vinculin (Thermo Fisher Scientific), both diluted 1:1000. Primary antibodies were detected using horseradish peroxidase-linked antirabbit (Agilent Dako), diluted 1:2000 and visualized using the enhanced chemiluminescent detection system (SuperSignal West Dura Extended Duration Substrate, Thermo Fisher Scientific).

Levels of PD-L1 expression were evaluated by densitometric analysis using the software available in the gel documentation system (Alliance, Uvitec Ltd.). Integrated optical density (IOD) was analyzed for each cell line and calculated after normalization of vinculin expression.

\section{Statistical Analysis}

Statistical analysis was performed with the Brown-Forsythe ANOVA test (Prism version 7, GraphPad Software) with Welch's correction, after performing normality and $\log$ normality tests.

\section{Results}

\section{Baseline Patient Characteristics}

Overall, 7 treatment-naive patients who underwent 
TABLE 1. Baseline patient characteristics

\begin{tabular}{|c|c|}
\hline & Value $(n=7)$ \\
\hline \multicolumn{2}{|l|}{ Demographic } \\
\hline Mean age $\pm S D$, yrs & $63.3 \pm 12.8$ \\
\hline Male sex & $6(85.7)$ \\
\hline \multicolumn{2}{|l|}{ Radiological } \\
\hline \multicolumn{2}{|l|}{ Location } \\
\hline Postcentral & $3(42.85)$ \\
\hline Temporoinsular & $4(57.15)$ \\
\hline Rt side & $4(57.15)$ \\
\hline Mean preop vol $\pm \mathrm{SD}, \mathrm{cm}^{3}$ & $38.85 \pm 20$ \\
\hline Corpus callosum involvement & $4(57.15)$ \\
\hline Ependymal involvement & $4(57.15)$ \\
\hline Necrotic cystic component & $5(71.42)$ \\
\hline Midline shift & $2(28.57)$ \\
\hline Supratotal resection on postop MRI & $7(100)$ \\
\hline \multicolumn{2}{|l|}{ Molecular } \\
\hline Ki-67 index $>20 \%$ & $7(100)$ \\
\hline IDH1 mutation & $0(0)$ \\
\hline MGMT mutation & $3(42.85)$ \\
\hline \multicolumn{2}{|l|}{ Clinical } \\
\hline \multicolumn{2}{|l|}{ Oncological protocol } \\
\hline Stupp & $5(71.42)$ \\
\hline Stupp + carmustine & $1(14.28)$ \\
\hline Radiotherapy only & $1(14.28)$ \\
\hline
\end{tabular}

IDH1 = isocitrate dehydrogenase 1; MGMT = 0-6-methylguanine-DNA methyltransferase.

Values represent the number of patients (\%) unless indicated otherwise. surgery for a histopathologically confirmed diagnosis of GBM at our two centers between October 2019 and March 2020 were included. Their baseline characteristics are reported in Table 1.

\section{GASC Isolation and Characterization}

Samples representative of the 3 regions of interest (ALA+, ALA PALE, and ALA-) were collected for each patient and histologically examined. ALA+ specimens were characterized by the appearance of a high-grade tumor; ALA PALE samples showed a gliotic tissue focally infiltrated; and ALA- specimens did not show recognizable tumor cells. Despite their different histological aspects, GASCs were efficiently isolated from each of the 3 regions without significant differences. Previous research conducted by our group had demonstrated that 1) phenotypic characterization of GASCs derived from the ALA+, ALA PALE, and ALA- regions was the same, even if GASCs derived from ALA- showed a reduced activation state; 2) gene expression was different in the GASCs isolated from the 3 areas (Fig. 3); and 3) PD-L1 was among the upregulated genes in GASCs derived from the ALA+ region. ${ }^{24}$

\section{Do GASCs Derived From Different ALA Regions Differentially Express PD-L1?}

Gene expression does not necessarily equate to protein expression. Our hypothesis was that GASCs derived from ALA+ would be able to selectively express PD-L1 in protein as well. Against this background, we assessed PD-L1 expression in the protein of GASC samples taken at different distances from the GBM core (ALA+, ALA PALE, and ALA-).

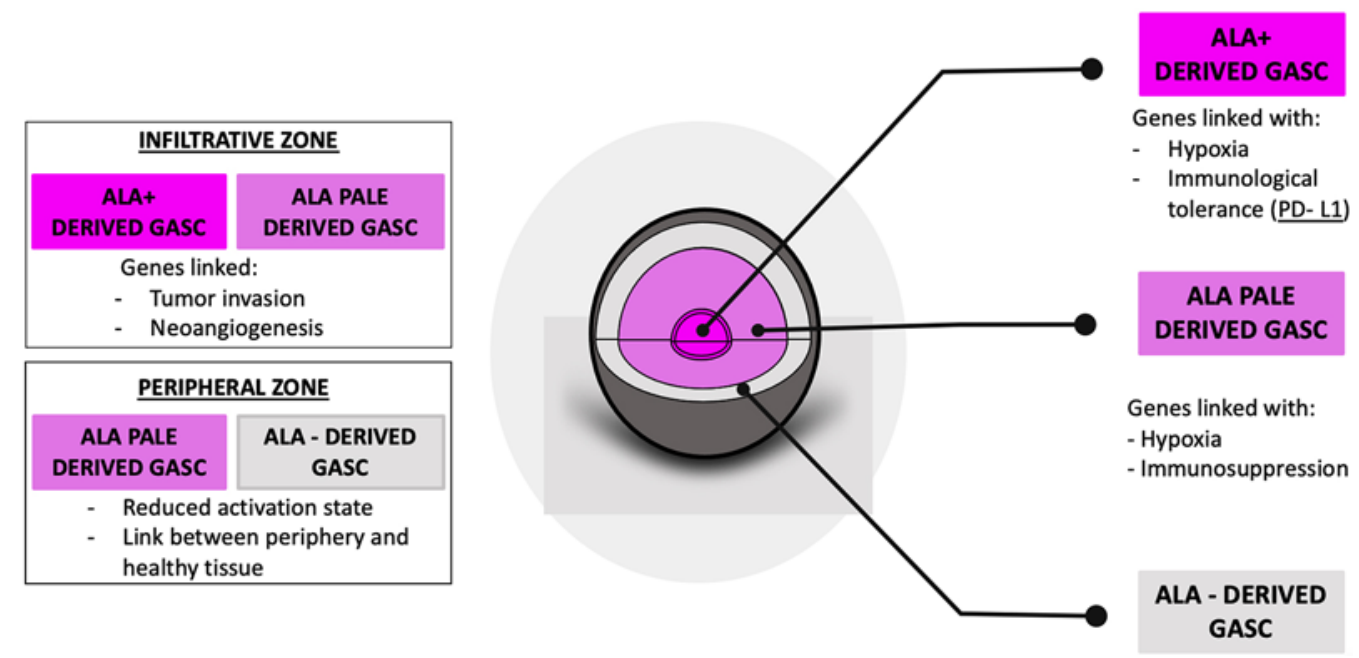

FIG. 3. Schematic representation of the findings of our previous research. Comparing GASCs of the different regions (ALA+vs ALA-, ALA+ vs ALA PALE, and ALA PALE vs ALA-), it was demonstrated that GASCs derived from the core (top right) preferentially expressed promoting hypoxia and immunological tolerance (PD-L1 included). GASCs derived from the infiltrative zone (core and pale; upper left) genes promoted invasion and neoangiogenesis. ALA PALE-derived GASCs were linked with hypoxia and immunosuppression (center right). Periphery-derived GASCs (ALA PALE and ALA-; lower left) formed the link between the tumor periphery and healthy tissue, despite having a reduced activation state. Counterintuitively, the last point could be potentially relevant from a therapeutic standpoint as the peripheral area is the least surgically treatable, while also the area from which both relapse and a resistance to second-line treatments originate. 

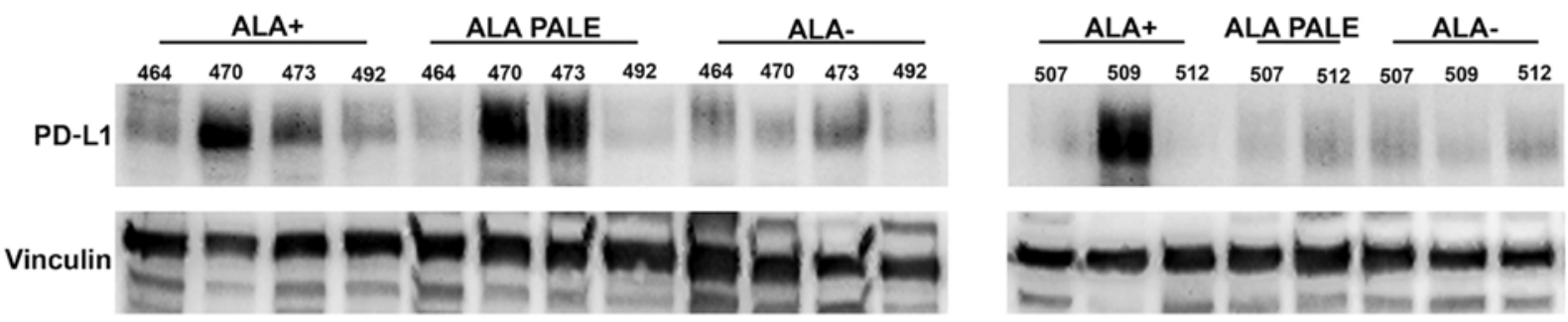

FIG. 4. Western blotting for PD-L1 protein expression on GASC cell lines isolated from the 3 sampling sites. Vinculin was used as the housekeeping protein. The analysis was performed on GBM samples obtained from 7 different patients.

For qualitative analysis, Western blotting for PD-L1 protein expression in GASC cell lines isolated from the 3 tumor regions was performed, with vinculin used as the housekeeping protein (Fig. 4). For quantitative analysis, the results of densiometric analysis were calculated as IOD after normalization of vinculin. Each assay was repeated 3 times.

Overall, the mean values \pm SD of PD-L1 expression in ALA+, ALA PALE, and ALA- were $1.12 \pm 1.14,0.89 \pm$ 0.63 , and $0.57 \pm 0.18$, respectively (Table 2 ).

The differentially expressed values of PD-L1 in GASCs sampled from the 3 areas were found to be significant ( $p$ $<0.05$ ) for 3 of 7 patients: patient S470 (ALA+ vs ALAand ALA PALE vs ALA-), patient S473 (ALA+ vs ALA PALE and ALA PALE vs ALA-), and patient S509 (ALA+ vs ALA-). In the latter case, an unpaired t-test (two-tailed) with Welch's correction was performed (Fig. 5). In these 3 patients, GASCs isolated at the level of the ALA PALE region, corresponding to the tumor periphery, showed a statistically significant PD-L1 overexpression compared with those cells isolated from the surrounding "healthy brain." As confirmation of this, the cumulative data showed no statistically significant differences in PD-L1 expression in the GASCs isolated from the 3 target areas.

To better emphasize and visualize these findings, results were reported as the fold induction of PD-L1 in ALA+ compared with ALA PALE, ALA+ compared with ALA-, and ALA PALE compared with ALA- (Fig. 6). Our analysis showed, for the first time, that GASCs express a constitutive level of PD-L1, contributing to the creation of an immunosuppressive environment; however, this level has a statistical significance, in terms of differential expression at infiltrative margins, only in those patients with a constitutive overexpression.

\section{Discussion}

Taking advantage of fluorescence-guided surgery, we exploited a human in vitro model of the glioma microenvironment, represented by GASCs. This approach allowed us to demonstrate, for the first time, that when moving from the ALA+ region (corresponding to the tumor core) toward the ALA PALE region (corresponding to its infiltrating front) to the ALA- region (beyond the infiltrating front as assessed by fluorescence) the PD-L1 expression profile of GASCs changed. Moreover, 5-ALA-guided surgery in combination with the study of GASCs made it possible to highlight that this crosstalk was not uniform throughout the tumor; infiltration and immune tolerance were particularly present in the infiltrating margins, which is the region from which the recurrence possibly originates. ${ }^{23}$

\section{PD-L1 Expression at the Protein Level in GASCs}

Our previous findings suggested that the infiltrative margin was directly related to the onset of relapse, with GASCs playing a key role in the area. This supported the existence of a bidirectional crosstalk between glioma cells and their surrounding microenvironment. (GASCs cooperate with the TME, inducing changes in its phenotypes and its ability to support tumor growth while it releases protumorigenic signals. $)^{24}$

As mentioned earlier, gene expression is not equivalent to protein expression. In normal conditions, PD-1 and PD-L1 engagement avoids a prolonged activation of the immune system, particularly related to autoimmunity processes. Initially, the dysregulation of this pathway, which also occurs in high-grade gliomas, has been proposed as a therapeutic target. Unfortunately, there were unsatisfactory results from those phase III clinical trials that directly targeted PD-1.

Based on this knowledge, a new approach that is focused on targeting PD-L1 expression in the general cancer cell population has quickly taken hold. ${ }^{7,25}$ Several studies demonstrated that higher expression of PD-L1 (at both the protein and mRNA levels) was linked to worse outcome; PD-L1 might expand and maintain immunosuppressive regulatory T cells, which in turn are associated with decreased survival in patients with gliomas. Nevertheless,

TABLE 2. Results of densiometric analysis to evaluate PD-L1 protein content in GASCs derived from the 3 regions of each patient

\begin{tabular}{rrrr}
\hline PtNo. & \multicolumn{1}{c}{ ALA+ } & ALA PALE & \multicolumn{1}{c}{ ALA- } \\
\hline S464 & $0.55 \pm 0.11$ & $0.47 \pm 0.05$ & $0.43 \pm 0.01$ \\
\hline S470 & $1.9 \pm 0.42$ & $1.89 \pm 0.07$ & $0.38 \pm 0.01$ \\
\hline S473 & $0.95 \pm 0.12$ & $1.5 \pm 0.06$ & $0.86 \pm 0.09$ \\
\hline S492 & $0.5 \pm 0.13$ & $0.59 \pm 0.15$ & $0.59 \pm 0.15$ \\
\hline S507 & $0.61 \pm 0.01$ & $0.4 \pm 0.07$ & $0.48 \pm 0.18$ \\
\hline S509 & $3.51 \pm 0.03$ & NA & $0.76 \pm 0.12$ \\
\hline S512 & $0.37 \pm 0.12$ & $0.53 \pm 0.07$ & $0.47 \pm 0.12$ \\
\hline Total & $1.12 \pm 1.14$ & $0.89 \pm 0.63$ & $0.57 \pm 0.18$ \\
\hline
\end{tabular}

$\mathrm{NA}=$ not available; $\mathrm{pt}=$ patient.

Values are presented as mean \pm SD. Each assay was repeated 3 times. 


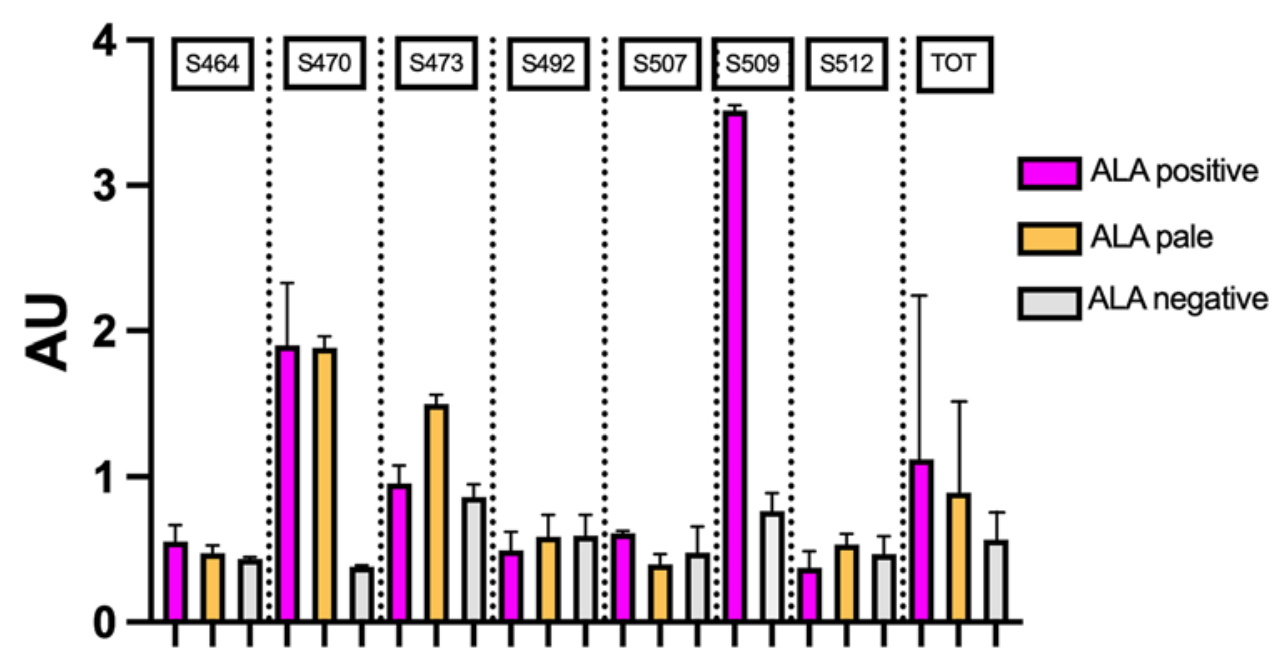

FIG. 5. Histograms showing results of the densiometric analysis performed to evaluate the PD-L1 protein content in GASCS derived from the 3 regions sampled from each patient. Values were calculated as the IOD value of PD-L1, after normalization of vinculin. Each assay was repeated 3 times. Statistical analysis was performed with the one-way ANOVA test. The total (TOT) reports the mean values of PD-L1 expression in the ALA+, ALA PALE, and ALA- regions of all patients. Data are presented as mean $\pm S D$. $A U=$ arbitrary units.

studies have shown heterogeneity of PD-L1 expression in tumors, with greater expression seen at the edges of the tumor compared with the core, which led to the analysis of regulatory mechanisms contributing to enriched PDL1. $., 6,18,19,26-28$

Given the therapeutic potential and promising therapies targeting the PD-1/PD-L1 axis in GBM, we decided to fur-

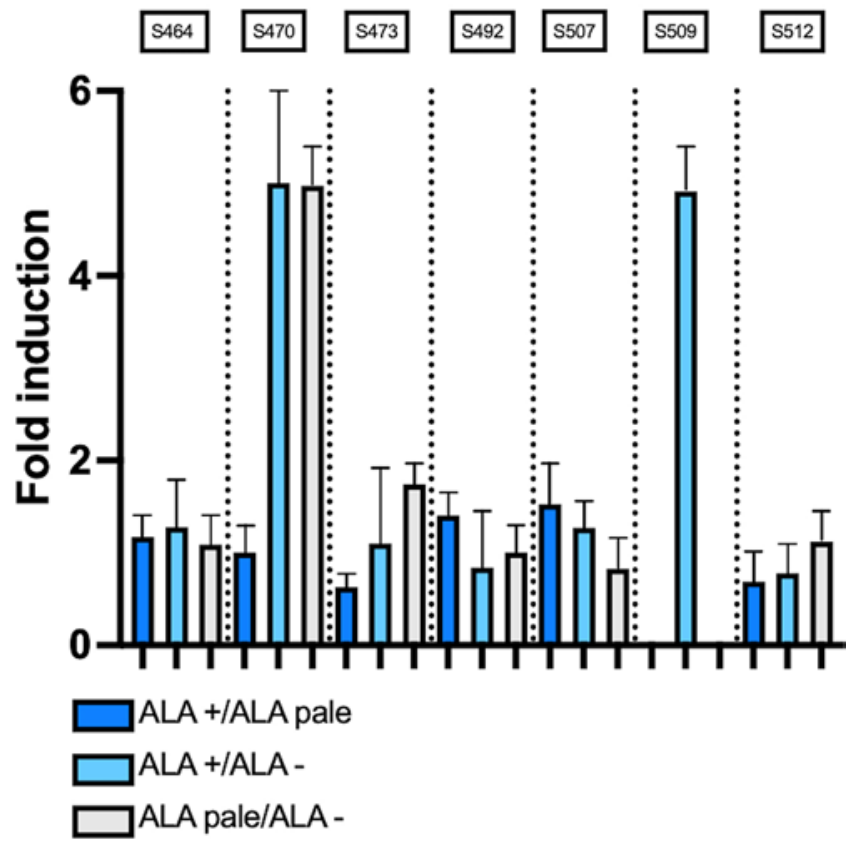

FIG. 6. Comparison of PD-L1 protein expression in GASCs isolated from the 3 sampling sites. Histograms showing results reported as the fold induction of PD-L1 in ALA+ compared with ALA PALE (dark blue), ALA+ compared with ALA- (light blue), and ALA PALE compared with ALA(gray) for each patient. ther explore the association between GASCs and this axis, in particular, because the expression of PD-L1 in GASC protein has never been described before and could have a potential therapeutic benefit.

Against this background, we assessed PD-L1 expression in samples taken at different distances from the GBM core (ALA+, ALA PALE, and ALA-). In 3 of the 7 patients analyzed (patients S470, S473, and S509), differential expression of PD-L1 was reported in GASCs isolated from ALA+ and ALA PALE versus the ALA- area $(\mathrm{p}<0.05)$ (Fig. 5). In these patients, GASCs were present in the tumor periphery, which corresponds to the infiltrating tissue, and, thus, were directly involved in immunosuppression pathways. In the remaining patients, GASCs derived from the ALA+ and ALA PALE areas did not express more PD-L1 than those derived from the ALA- area $(\mathrm{p}>0.05)$. This confirmed that even in a small sample like ours, the use of immunotherapy requires a careful selection of patients upstream. As confirmation of this, the cumulative data showed no statistically significant differences in PDL1 expression in GASCs isolated from the 3 target areas.

To summarize, our analysis showed that GASCs express a constitutive level of PD-L1, contributing to the creation of an immunosuppressive environment, and that this level has a statistical significance, in terms of differential expression at infiltrative margins, only in those patients with a constitutive overexpression.

\section{What Is Next? A Spatial-Temporal Perspective}

The reported data open several perspectives for the future treatment of gliomas, both "spatially" and "temporally." To define a spatial direction of what could be a future implication of this study is to emphasize that tumor boundaries, as recognized by 5-ALA, are not only surgical margins but also infiltration identifiers.

Our analysis was focused on a human in vitro model of the glioma microenvironment, represented by GASCs. 
However, we have seen how these cells actively interact with other players of the TME, which is a potential therapeutic target for GBM treatment as well. Therefore, the infiltrating margins of the pathology identified in vivo by 5-ALA should be recreated as closely as possible in vitro to ensure models that have a direct clinical implication. ${ }^{13}$ To do so, instead of recreating the complex brain matrix by controlled fabrication, cells can also be seeded into a matrix and stimulated to develop into organoids. In addition, the role of a locoregional therapy that directly targets those proteins differentially expressed in the infiltrating margins deserves further investigation.

To define a temporal direction of what could be a future implication of this study is to emphasize that both multiple sampling and tumor heterogeneity can vary under the selective pressure of therapies. Therefore, it would be interesting to evaluate tumor heterogeneity not only spatially (ALA+, ALA PALE, and ALA-) but also temporally (longitudinal samplings within the same patient in the 3 areas at the time of recurrence). This would allow for greater understanding of how the microenvironment changes over time as well as for assessment of the reliability of in vitro models in predicting therapeutic responses. ${ }^{29-31}$

\section{Limitations}

Despite that analyzing GASCs in invasion niches in GBM, with specific reference to the immunosuppressive status, gave us important insight into the role of infiltrating margins in high-grade glioma, the results of this study are not devoid of limitations. First is the small sample size of our study, which makes it a preliminary one. In addition, our sample is homogeneous in terms of race, ethnicity, and sex. Recent studies have shown how ethnic differences are relevant with regard to immunotherapy response in general and how sex-based differences may play a role in immunotherapy for gliomas. Hence, this study should be regarded as a preliminary study of feasibility that needs further investigation and larger samples to be validated. A second limitation derives from the sampling area; this holds true for the ALA PALE region especially. There is no universally defined fluorescence grading system, and there may have been operator-dependent inhomogeneity between the two centers. Third, and importantly, PD-L1 protein expression was evaluated only at the cellular level (in GASCs isolated from the 3 sampling areas), not histologically, in our study. Recently, the overexpression of PD-L1 in high-grade glioma tissue compared with healthy brain has been demonstrated; it would be interesting to investigate its expression not only in bulk-resected tissue but also in relation to the infiltrative margins (corresponding to the ALA PALE region). ${ }^{32}$

\section{Conclusions}

Our analysis showed, for the first time, that GASCs express a constitutive level of PD-L1 and that PD-L1 expression in GASCs is not uniform among patients or within the same patient. Differential PD-L1 expression in the protein of GASCs that were isolated from different tumor areas made it possible to underline the crosstalk between tumor cells and the microenvironment, which is not consistent throughout the tumor, and to point out how invasiveness properties might be differentially supported by the different regions. Our analysis made it possible to highlight the key role of the TME at the infiltrating margin. This opens interesting perspectives for the future treatment of gliomas, particularly those linked to the advancement of immunotherapy at infiltrating margins.

\section{References}

1. GBD 2016 Brain and Other CNS Cancer Collaborators. Global, regional, and national burden of brain and other CNS cancer, 1990-2016: a systematic analysis for the Global Burden of Disease Study 2016. Lancet Neurol. 2019;18(4): 376-393.

2. Tan AC, Ashley DM, López GY, Malinzak M, Friedman HS, Khasraw M. Management of glioblastoma: state of the art and future directions. CA Cancer J Clin. 2020;70(4):299-312.

3. Holland EC. Glioblastoma multiforme: the terminator. Proc Natl Acad Sci U S A. 2000;97(12):6242-6244.

4. Goodwin CR, Laterra J. Neuro-oncology: unmasking the multiforme in glioblastoma. Nat Rev Neurol. 2010;6(6):304305.

5. Wenger KJ, Wagner M, You SJ, et al. Bevacizumab as a lastline treatment for glioblastoma following failure of radiotherapy, temozolomide and lomustine. Oncol Lett. 2017;14(1): 1141-1146.

6. Rath BH, Wahba A, Camphausen K, Tofilon PJ. Coculture with astrocytes reduces the radiosensitivity of glioblastoma stem-like cells and identifies additional targets for radiosensitization. Cancer Med. 2015;4(11):1705-1716.

7. Reardon DA, Brandes AA, Omuro A, et al. Effect of nivolumab vs bevacizumab in patients with recurrent glioblastoma: the CheckMate 143 phase 3 randomized clinical trial. JAMA Oncol. 2020;6(7):1003-1010.

8. Tawbi HA, Forsyth PA, Algazi A, et al. Combined nivolumab and ipilimumab in melanoma metastatic to the brain. $N$ Engl J Med. 2018;379(8):722-730.

9. Tawbi HAH, Forsyth PAJ, Hodi FS, et al. Efficacy and safety of the combination of nivolumab (NIVO) plus ipilimumab (IPI) in patients with symptomatic melanoma brain metastases (CheckMate 204). J Clin Oncol. 2019;37(15 Suppl):9501.

10. Goldberg SB, Schalper KA, Gettinger SN, et al. Pembrolizumab for management of patients with NSCLC and brain metastases: long-term results and biomarker analysis from a non-randomised, open-label, phase 2 trial. Lancet Oncol. 2020;21(5):655-663.

11. Cloughesy TF, Mochizuki AY, Orpilla JR, et al. Neoadjuvant anti-PD-1 immunotherapy promotes a survival benefit with intratumoral and systemic immune responses in recurrent glioblastoma. Nat Med. 2019;25(3):477-486.

12. Taggart D, Andreou T, Scott KJ, et al. Anti-PD-1/antiCTLA-4 efficacy in melanoma brain metastases depends on extracranial disease and augmentation of $\mathrm{CD}^{+} \mathrm{T}$ cell trafficking. Proc Natl Acad Sci U S A. 2018;115(7):E1540-E1549.

13. Wolf KJ, Chen J, Coombes J, Aghi MK, Kumar S. Dissecting and rebuilding the glioblastoma microenvironment with engineered materials. Nat Rev Mater. 2019;4(10):651-668.

14. Chuntova P, Chow F, Watchmaker PB, et al. Unique challenges for glioblastoma immunotherapy-discussions across neuro-oncology and non-neuro-oncology experts in cancer immunology. Meeting Report from the 2019 SNO ImmunoOncology Think Tank. Neuro Oncol. 2021;23(3):356-375.

15. Bourkoula E, Mangoni D, Ius T, et al. Glioma-associated stem cells: a novel class of tumor-supporting cells able to predict prognosis of human low-grade gliomas. Stem Cells. 2014;32(5):1239-1253.

16. Cesselli D, Beltrami AP, Pucer A, et al. Human low-grade 
glioma cultures. In: Duffau H, ed. Diffuse Low-Grade Gliomas in Adults. Springer; 2017:137-163.

17. Caponnetto F, Beltrami AP, Ius T, Skrap M, Cesselli D. Diffuse low-grade glioma associated stem cells. In: Duffau H, ed. Diffuse Low-Grade Gliomas in Adults. Springer; 2017:151-172.

18. Litak J, Mazurek M, Grochowski C, Kamieniak P, Roliński J. PD-L1/PD-1 axis in glioblastoma multiforme. Int J Mol Sci. 2019;20(21):5347.

19. Hsu JM, Xia W, Hsu YH, et al. STT3-dependent PD-L1 accumulation on cancer stem cells promotes immune evasion. Nat Commun. 2018;9(1):1908.

20. Hadjipanayis CG, Stummer W. 5-ALA and FDA approval for glioma surgery. J Neurooncol. 2019;141(3):479-486.

21. Traylor JI, Pernik MN, Sternisha AC, McBrayer SK, Abdullah KG. Molecular and metabolic mechanisms underlying selective 5-aminolevulinic acid-induced fluorescence in gliomas. Cancers (Basel). 2021;13(3):580.

22. Dadario NB, Khatri D, Reichman N, Nwagwu CD, D'Amico RS. 5-aminolevulinic acid-shedding light on where to focus. World Neurosurg. 2021;150:9-16.

23. Müther M, Koch R, Weckesser M, Sporns P, Schwindt W, Stummer W. 5-aminolevulinic acid fluorescence-guided resection of 18F-FET-PET positive tumor beyond gadolinium enhancing tumor improves survival in glioblastoma neurosurgery. 2019;85(6):E1020-E1029.

24. Manini I, Caponnetto F, Dalla E, et al. Heterogeneity matters: different regions of glioblastoma are characterized by distinctive tumor-supporting pathways. Cancers (Basel). 2020; 12(10):2960.

25. Kelly WJ, Giles AJ, Gilbert M. T lymphocyte-targeted immune checkpoint modulation in glioma. J Immunother Cancer. 2020;8(1):e000379.

26. Scheffe TB, Grave N, Vargas P, Diz FM, Rockenbach L, Morrone FB. Immunosuppression in gliomas via PD-1/PD-L1 axis and adenosine pathway. Front Oncol. 2021;10:617385.

27. Yao Y, Tao R, Wang X, Wang Y, Mao Y, Zhou LF. B7-H1 is correlated with malignancy-grade gliomas but is not expressed exclusively on tumor stem-like cells. Neuro Oncol. 2009;11(6):757-766.
28. Wilmotte R, Burkhardt K, Kindler V, et al. B7-homolog 1 expression by human glioma: a new mechanism of immune evasion. Neuroreport. 2005;16(10):1081-1085.

29. Xue S, Hu M, Iyer V, Yu J. Blocking the PD-1/PD-L1 pathway in glioma: a potential new treatment strategy. J Hematol Oncol. 2017;10(1):81.

30. Fisher JP, Adamson DC. Current FDA-approved therapies for high-grade malignant gliomas. biomedicines. 2021;9(3):324.

31. Di Mascolo D, Palange AL, Primavera R, et al. Conformable hierarchically engineered polymeric micromeshes enabling combinatorial therapies in brain tumours. Nat Nanotechnol. 2021;16(7):820-829.

32. Hölzl D, Hutarew G, Zellinger B, et al. Integrated analysis of programmed cell death ligand 1 expression reveals increased levels in high-grade glioma. J Cancer Res Clin Oncol. 2021; 147(8):2271-2280.

\section{Disclosures}

Financed by Progetto Ministero della Salute, Giovani Ricercatori 2016 GR-2016-02364678. Application of GLIADEL wafers (BCNU, carmustine) followed by temozolomide and radiotherapy in patients with high-grade glioma: a precision medicine based on molecular landscape. Principal investigator: Tamara Ius.

\section{Author Contributions}

Conception and design: Menna, Manini. Acquisition of data: Manini. Analysis and interpretation of data: Menna, Della Pepa. Drafting the article: Menna, Ius, Della Pepa. Critically revising the article: Menna, Ius, Della Pepa. Reviewed submitted version of manuscript: Ius. Statistical analysis: Manini, Della Pepa. Study supervision: Cesselli, Skrap, Olivi, Ius, Della Pepa.

\section{Correspondence}

Grazia Menna: Institute of Neurosurgery, Fondazione Policlinico Universitario Agostino Gemelli IRCCS, Catholic University, Rome, Italy.mennagrazia@gmail.com. 